\title{
Prevención de Preeclampsia y Eclampsia: Mitos y Realidades.
}

\author{
Andrés Sarmiento, MD.*; Walter Pinzón, MD.**
}

\section{RESUMEN}

Se practica una revisión de los conceptos actuales en la prevención de preeclampsia y tratamiento de la Eclampsia. Se analizan los diferentes estudios disponibles en la literatura obstétrica acerca de la validez clínica de cada una de las propuestas terapéuticas. Se concluye que ninguna de las alternativas presenta resultados clínicos como para considerar su uso rutinario. Se comentan los resultados en el escenario de la practica obstétrica actual. Se insiste en la necesidad de continuar en líneas de investigación para el discernimiento de la etiología de la preeclampsia; se presume que solo así se podría acercar a respuestas terapéuticas validas.

Palabras claves: Preclampsia, eclampsia, calcio, aspirina, nimodipina.

\section{SUMMARY}

A review of actual concepts in the prevention of preeclampsia and treatment of eclampsia is practiced. The different studies are analized and their clinical results. We conclude that none of the different therapeutical options proposed are valid for their clinical rutinary use. Comments upon the results within our clinical escenario are done. We insist in the need for mayor research in the study of the etiology of preclampsia; maybe that will be the only way to introduce therapeutic options with real clinical value.

Key words: Preclampsia, eclampsia, calcium, aspirine, nimodipine.

\section{Introducción}

La preclampsia es un desorden multisistémico de origen desconocido y propio de la especia humana. Dependiendo de la población, grupo étnico o región que se analice, complica a aproximadamente un $15-10 \%$ de todos los embarazos. En nuestro Hospital la incidencia de la enfermedad es de $6.8 \%$.

A pesar de ser esta entidad una generadora importante de morbimortalidad materno-fetal (aumenta 5 veces la mortalidad perinatal) su etiología aún no se conoce. La información científica disponible a la fecha ha demostrado cómo una defectuosa respuesta vascular a la placentación ocurrida durante el primer trimestre es el defecto primario que desencadena el cuadro clínico. El proceso fisiopatológico de placentación incluye dos invasiones trofoblásticas que inicialmente compromete los segmentos deciduales de las arterias espirales para luego extenderse hasta el tercio interno del miometrio. Este fenómeno transforma un territorio vascular de alta resistencia a uno de alta capacitancia y baja resistencia y que característicamente exhibe una pobre respuesta a sustancias vasopresoras. En pacientes preeclámpticas se ha demostrado que la segunda invasión trofoblástica no se lleva a cabo resultando en un

\footnotetext{
* Coordinador Académico Unidad de Medicina Materno-Fetal, Hospital Simón Bolívar. Profesor Universidad El Bosque. Bogotá.

Residente IV de Ginecobstetricia, Universidad El Bosque, Hospital Simón Bolívar, Bogotá.
}

territorio placentario con vasoconstricción, sensibilidad aumentada a aminas y pépticos vasopresores y a un flujo uteroplacentario reducido.

El limitado entendimiento de su etiología ha influido en forma importante en el fracaso para establecer medidas preventivas efectivas y poder establecer que pacientes desarrollarán la enfermedad. Una variedad importante de pruebas clínicas, bioquímicas, biofísicas y hemodinámicas han sido propuestas para la identificación de la paciente que desarrollará preeclampsia. Lamentablemente la mayoría gozan de una pobre sensibilidad diagnóstica y no tienen aplicabilidad clínica. Un gran número de estudios clínicos se han llevado a cabo tratando de disminuir la incidencia de preeclampsia y/o su severidad. La gran mayoría de ellos han mostrado resultados desalentadores.

A continuación haremos una relación de las propuestas terapéuticas disponibles en la actualidad para la prevención de preeclampsia y eclampsia. Pretendemos hacer un análisis crítico muy objetivo basados en la evidencia científica existente hasta la fecha, matizado por nuestra propia experiencia en la Unidad de Medicina MaternoFetal del Hospital Simón Bolívar.

\section{Prevencion de preeclampsia}

\section{Aspirina}

Se ha establecido que en la preclampsia existe un disbalance en el metabolismo prostaglandínico que favo- 
rece la relación tromboxano $A 2$ (TXA2) / prostaciclina (PGI2). Estos dos eicosanoides tienen efectos opuestos; mientras el TxA2 es un potente vasoconstrictor, la PGI2 es un vasodilatador e inhibidor de la agregación plaquetaria. Asimismo el oxido nítrico, como un producto del endotelio vascular, ha sido propuesto como un factor que reduce el tono vascular y evita la adhesión y agregación plaquetarias. Basados en estos fenómenos fisiológicos la aspirina (ASA) fue propuesta como un profiláctico que intervendría activamente en la prevención de la preeclampsia. Su acción inhibitoria sobre la ciclooxigenasa y la producción de TxA2 restablecería el balance TxA2 / PGI2. Así mismo se ha propuesto que bloquea la exagerada respuesta a la angiotensina evidenciada en preeclámpticas.

En 1979, Crandon publicó el primer estudio acerca de la efectividad de la ASA en la prevención de preeclampsia. Durante los siguientes años se publicaron un buen número de estudios prospectivos y randomizados acerca de la eficacia de este medicamento. En 1985, se publicó el primer estudio prospectivo randomizado en el cual ptes. con una pobre historia obstétrica o hipertensión arterial crónica eran designadas a recibir ASA (150mgs/día) vs ningún tratamiento. El estudio incluyó 93 pacientes y demostró un claro beneficio (0 vs $13 \%$ ) a favor de la ASA en la prevención de preclampsia. En 1993 se publicó el primer metaanálisis por parte de Sibai donde se concluía acerca de los beneficios de la ASA en la prevención de HTA gestacional, preeclampsia y RCIU. Se demostró una reducción promedio de preeclampsia del $70 \%$.

A pesar de la euforia que produjeron estos resultados iniciales, al practicar un análisis retrospectivo de estos estudios se empezaron a evidenciar la presencia de algunos vicios estadísticos. Un buen número de trabajos tenían una muestra poblacional limitada y en otros existían deficiencias importantes en el momento de definir la preeclampsia. Por ejemplo, en algunos estudios se diagnosticaba la preeclampsia sobre la base de proteinuria cualitativa. Muchos de los estudios concluían demostrando una disminución en la incidencia de preeclampsia, sin ninguna repercusión en la morbimortalidad perinatal. Un buen número de estudios solamente incluía población seleccionada de alto riesgo. Finalmente, fue evidente cómo muchos estudios pequeños con resultados desfavorables nunca fueron tenidos en cuenta para su publicación y mucho menos para hacer parte del análisis estadístico.

Desde 1993 hasta la fecha se han publicado algunos pequeños estudios aislados y 8 grandes trabajos multicéntricos que en total engloban algo más de 27.100 pacientes. Solo uno (1) de los estudios demostró un beneficio en la prevención de preeclampsia con la ASA. Ningún trabajo demostró un beneficio en el resultado final perinatal. Un clásico estudio multicéntrico como el CLASP en 9.364 gestantes demostró una reducción en la incidencia de preclampsia de tan solo un $12 \%$, hallazgo que no demostró trascendencia clínica. Así mismo evidenció una tendencia a la reducción de preclampsia únicamente en pacientes con parto pretérmino. Los autores concluyeron que se amerita administrar la ASA únicamente en pacientes con factores de riesgo muy claros para parto pretérmino.

Quizás el trabajo más contundente publicado en los últimos años es el presentado por Caritis. A nuestro juicio es el estudio más completo y que mayor información aporta en el entendimiento del verdadero papel de la ASA en la prevención de la preclampsia. Fue un estudio multicéntrico que cobijó trece centros médicos. Incluyó 2.539 pacientes que presentaban los siguientes factores de riesgo: hipertensión arterial crónica, diabetes gestacional, embarazo múltiple o antecedente de preeclampsia. La población fue randomizada en 2 grupos administrando $60 \mathrm{mgs}$ de ASA vs placebo, a partir de las 13-26 semanas. No se demostró una reducción significativa en la incidencia de preclampsia (18 vs $20 \%$ ). Así mismo no existieron diferencias con relación a morbimortalidad perinatal, bajo peso al nacer o parto pretérmino.

Los autores concluyen el trabajo haciendo una comparación entre los estudios iniciales con grupos poblacionales reducidos (menos de 200 pacientes) que sugerían una reducción promedio de $8.2 \%$ con estudios recientes (con más de 200 pacientes) que indican reducción promedio de preeclampsia de tan solo un $0.9 \%$, valor que no guarda validez clínica.

Los diferentes trabajos convergen en asegurar que la ASA a las dosis indicadas parece ser segura para el embarazo. No afecta la PGI2 fetal, la síntesis o función plaquetaria, el gasto urinario del neonato, la función cardíaca y en general el resultado final perinatal.

Basados en la información disponible a la actualidad no parece existir una justificación para el uso de la ASA en la prevención de la preclampsia. Luego de revisar los diferentes trabajos y decantados los conceptos allí expuestos se podrían hacer una serie de especulaciones:

Quizás la administración de ASA no debe hacerse indiscriminadamente a pacientes de alto o bajo riesgo. Alguna información sugiere que el enfoque ideal debería ser su dosificación únicamente a población de alto riesgo que además presente pruebas de selección anormales como flujo uterino anormal.

Sin embargo, el consenso médico-científico general afirma que dada la evidencia disponible en la actualidad no tiene sentido insistir con nuevos protocolos de ASA y embarazo. La mayoría de grupos de investigación hoy en día dirigen sus esfuerzos hacia el entendimiento de la etiología de la preeclampsia y de los mecanismos de anormalidades multisistémicas.

Los estudios acerca del tema no deben solamente demostrar disminución en la presión arterial por la administración de un fármaco para ser considerados exitosos. La tensión arterial es un signo clínico de limitada trascendencia dentro del proceso fisiopatológico de la preclampsia, no es la enfermedad. Los estudios han debido y deben probar disminución en la morbimortalidad perinatal para ser considerados válidos.

\section{Suplementacion con calcio}

Durante la pasada década ha existido un creciente interés por el papel que puede desempeñar el calcio en la prevención de la preeclampsia. La observación epidemiológica que poblaciones con alta ingesta de calcio (Etíopes o Guatemaltecos vs Thailandeses y Japoneses) en sus dietas tenían una baja incidencia de preclampsia, 
motivó a múltiples estudios del papel de la suplencia de calcio en la prevención de la enfermedad. Así mismo, la presencia de hipocalciuria en la preeclampsia pareció ser una evidencia mas de la validez de la observación.

El proceso fisiopatológico exacto no ha sido dilucidado aún, parece ser que la hipocalcemia estimula un aumento en la concentración de hormona paratiroidea y/o de renina, lo que aumenta la reabsorción de calcio a nivel renal. El aumento en las concentraciones séricas de hormona paratiroidea producen un ingreso del calcio a las células del músculo liso vascular con aumento en su tono y en la respuesta a presores. Así mismo se demostraron altas concentraciones de calcio en eritrocitos y plaquetas con pacientes con preeclampsia.

El papel del calcio en la prevención de preeclampsia ha sido sujeto de análisis en por lo menos 12 estudios publicados a la fecha. Lamentablemente la metodología de los estudios varía en forma importante: difiere en los grupos poblacionales, edad gestacional al ingreso, dosis, tamaño de la muestra y dosis de calcio, y definición de preeclampsia e hipertensión. De los doce estudios, cinco no tienen grupo control con placebo, no definen preeclampsia o utilizan calcio en combinación con otras drogas.

Si se analizan los siete estudios restantes publicados en la literatura, aquellos publicados por López Jaramillo tienen una baja casuística y presentan una incidencia de preeclampsia exageradamente alta para el grupo placebo (23.5\%)! ! Dos de Villar y Sánchez Ramos tienen una casuística realmente baja. El estudio de Levine es el que más casuística aporta: 4.589 pacientes sanas fueron randomizadas a recibir 2 gramos de calcio día o placebo, iniciando entre las semanas $13-26$ (2.295 vs 2.294). El resultado demostró una incidencia de preeclampsia de 6.9 (grupo de calcio) vs 7.3 (grupo control) y de hipertensión gestacional de 15 (Calcio) vs 17 (Control). La dosificación no demostró tampoco prevención de parto pretérmino o disminución de complicaciones perinatales. Se postularon posibles complicaciones como litiasis renal materna (si de base existe una tendencia al aumento en la absorción de calcio) y de hipocalcemia fetal.

El análisis objetivo de la información científica disponible no parece justificar la administración de calcio en la prevención de preeclampsia. O por lo menos asílo concluyen los diferentes autores que adelantaron los estudios referidos. El Instituto Nacional de Salud de los EEUU en un boletín técnico de 1997 determinó que dada la evidencia científica disponible no se justifica utilizar calcio en la prevención de preeclampsia.

\section{Acidos grasos polinsaturados}

El primer reporte de la utilidad de los ácidos grasos polinsatuturados (AGP) data de 1942. En 1990, Olsen en el British Medical Journal publica una casuística de 5.000 pacientes quienes recibieron vitaminas, minerales y aceite de hígado de jabalí. Los resultados reportaron una disminución en la incidencia de preeclampsia y parto pretérmino. Durante la última década múltiples estudios se han ocupado del tema con resultados desfavorables. El beneficio teórico del aceite de pescado radica en su contenido de cadenas largas de ácidos grasos n-3 polinsaturados :

$$
\begin{aligned}
& \text { Eicosapentaenoico (EPA) } \\
& \text { Docosa hexoenoico (DHA) } \\
& \text { Docosapentaenoico (DPA) }
\end{aligned}
$$

Estos ácidos inhiben la producción plaquetaria de TxA2 sin afectar la producción endotelial de PGI2, lo que conlleva a una estado de vasodilatación con reducción en la agregación plaquetaria.

La mayoría de estudios al respecto son de origen europeo y la gran mayoría han sido publicados en la Gran Bretaña. Información reciente de 3 estudios randomizados no demuestra beneficios con la suplementación de aceite de pescado. Los estudios de Bulstra (1994), Onwude (1995) (únicamente en mujeres de alto riesgo: 113 con aceite de pescado / $13 \%$ de preeclampsia vs 119 casos de placebo / $15 \%$ de preclampsia) y Saldig (1996) (266 mujeres sanas con aceite de pescado - 0 casos de preclampsia vs 131 placebo 1 caso de preclampsia), son conclusivos. Un estudio Europeo multicéntrico conducido por Dekker que comparó aceite de pescado vs aceite de oliva, falló asimismo en demostrar beneficios.

\section{Conclusiones}

La preclampsia sigue siendo una fuente importante de morbimortalidad materna y fetal. De los abordajes preventivos propuestas a la fecha, si bien algunos han sugerido disminución en la incidencia de hipertensión y/o preclampsia, no han demostrado reducción global de la morbimortalidad perinatal. El análisis objetivo de la información científica disponible sugiere que mientras que no se tenga mayor entendimiento de la etiología de la preclampsia difícilmente se podrán emprender estrategias claras de prevención con resultados clínicos determinantes y contundentes.

\section{Prevención de eclampsia}

Según estadísticas Americanas se ha estimado que la incidencia actual de eclampsia es de aproximadamente 0.56 por cada 1000 partos. Desafortunadamente los esquemas terapéuticos actuales que se basan en su mayor parte en la utilización de sulfato de magnesio no aseguran una prevención absoluta. Se ha estimado que la incidencia de convulsiones en la paciente tratada con sulfato oscila entre el $0.3 \%$ y el $4.3 \%$.

Las teorías mediante las cuales se han explicado las convulsiones en la paciente con preclampsia severa son :

- Vasoespasmo cerebral: éste ha sido demostrado mediante angiografía, T.A.C. y doppler intracraneano. Mediante éste último se ha demostrado que en la preclampsia severa la velocidad de flujo en la cerebral media está aumentada en forma importante.

- Encefalopatía hipertensiva: es la teoría más usualmente reconocida por internistas y neurólogos pero históricamente no compartida por los obstetras. En el estudio histopatológico se demuestra necrosis fibrinoide, microtrombos de fibrina, microinfartos y hemorragias. Aún cuando siempre se ha asociado a la vasoconstricción cerebral con la etiología de la preeclampsia, quizás existe 
cabida a considerar también los fenómenos de sobreperfusion propios de la encefalopatía hipertensiva. Esto podría explicar en parte las fallas de la terapia medicamentosa en el tratamiento de la eclampsia. Se ha demostrado como algunos vasos como la arteria cerebral media y otros vasos que irrigan los lóbulos occipitales no se vasocontriñen ante el fenómeno de hipertensión. Esta sobreperfusion podría ser la causante de la signología clínica propia de una encefalopatía hipertensiva.

Por lo tanto la eclampsia podría corresponder etiológicamente a dos fenómenos bien diferentes :

- Hipoperfusión secundaria al vasoespasmo, aún en la presencia de hipertensión arterial sistémica severa.

- Hiperperfusión debida a la pérdida de mecanismos protectores de autorregulación arterial.

De ser ciertas estas dos teorías estaríamos acercándonos a una respuesta a las fallas en la prevención de la eclampsia. Se especula que el enfoque terapéutico para una etiología no implicaría a la otra y viceversa; haciendo factible los fracasos terapéuticos que se evidencian hoy en día.

La droga de elección para el manejo de eclampsia ha sido y aún es el sulfato de magnesio. La vigencia de esta afirmación está debidamente sustentada por estudios recientes como el publicado por The Lancet (1995) donde un estudio multicéntrico internacional (en el que participa La Universidad del Valle-Colombia) demostró contundentemente cómo el sulfato aventaja al diazepam y a la fenitoína en cuando a efectividad terapéutica en términos de evitar recurrencia de convulsiones. Gracias a los resultados determinantes de éste estudio, el sulfato de magnesio está siendo nuevamente utilizado en muchos países y escenarios clínicos donde se le manejaba con reserva y escepticismo.

El mecanismo de acción del sulfato ha sido objeto de controversia. La información científica disponible a la fecha se puede resumir así:

1. Vasodilatación de los vasos cerebrales: Existe una oposición a la vasoconstricción arterial mediada por el calcio. Este fenómeno ha sido demostrado mediante doppler transcraneal y de la arteria retiniana. El índice de pulsatibilidad en las arterias cerebral media y orbitales se ve disminuido por el uso del sulfato, mejorando la perfusión vascular y revirtiendo la vasoconstricción cerebral.

2. Antagonismo al aumento en la concentración de calcio mediada por fenómenos de isquemia.

Esta demostración ha soportado la evidencia clínica de la limitada utilidad de la fenitoína. Este medicamento no tiene ningún influjo sobre la vasoconstricción cerebral y por ende no se muestra tan efectivo como el sulfato. La información así mismo soporta la teoría que la eclampsia no obedece a la activación de un foco epiléptico sino a aberraciones en los mecanismos de control vascular. De la misma manera el mecanismo de acción del sulfato no parece ser bloqueo neuromuscular o control aislado de los disparos de activación neuronal.

3. Efecto vasodilatador sistémico. A pesar que el sulfato no ha sido considerado como droga antihipertensiva, recientemente se ha demostrado sus propiedades de relajación vascular sistémica. Esta acción ha sido en parte explicada por su antagonismo con el calcio, pero también han sido propuestos un aumento en los niveles de monofosfato cíclico de guanosina, cambios en el sistema renina angiotensina y disminución en los niveles de endotelina 1. Es muy interesante anotar que el sulfato de magnesia tiene una acción diferente en la paciente embarazada que en la no embarazada, asícomo en la preeclámptica y en aquella con actividad uterina pretérmino. Se ha demostrado que el sulfato tiene un efecto hipotensor en la paciente hipertensa más no en la normotensa. Así mismo, otros estudios han demostrado cómo el efecto de vasodilatación se observa preferencialmente en pacientes preeclámpticas mientras en pacientes con actividad uterina pretérmino éste efecto es mínimo.

La conclusión de los trabajos parece establecer que el sulfato de magnesio exclusivamente en la paciente preeclámptica (no en casos de actividad uterina pretérmino) provoca una reducción sostenida de la resistencia vascular sistémica con un aumento en el índice cardíaco. Estos datos han tenido una correlación clínica. En un estudio reciente de 1.000 pacientes con preeclampsia severa (tensión arterial mayor a 160/110), un $40 \%$ no requirieron adicionar antihipertensivo luego de iniciar la infusión de sulfato.

Basados en la anterior información se puede hipotetizar que en la prevención de la eclampsia existiría cabida para la alternativa terapéutica de usar antihipertensivo puros con propiedades de vasodilatación cerebral, obviando el sulfato de magnesio. El uso de antihipertensivos de acción sistémica podría disminuir aún más la perfusión en un territorio cerebral ya hipoperfundido por vasoconstricción y agravar el problema. Drogas como el labetalol (inotrópico negativo más vasodilatador) para la paciente con encefalopatía hipertensiva podría ser un enfoque terapéutico viable.

Un medicamento propuesto para éste propósito es la nimodipina, un bloqueador de los canales de calcio, vasodilatador selectivo de la circulación cerebral y que viene siendo utilizado por neuróloges para revertir la vasoconstricción en pacientes con hemorragia subaracnoidea. Se trata de una medicación de mínimos efectos secundarios, bajo costo y administración oral. Su uso alternativo al sulfato obviaría los riesgos de depresión respiratoria, titulación de niveles séricos, engorrosa administración y necesidad de vigilancia médica permanente propios de éste. La nimodipina tiene un tremendo efecto vasodilatador sobre la microcirculación cerebral; efecto considerado hoy en día como clave en el discernimiento etiológico de la eclampsia.

Recientemente se presentó la primera experiencia del uso clínico de la nimodipina como alternativa al sulfato. Ciento diez pacientes con preeclampsia severa recibieron nimodipina vs. un grupo control de 108 quienes recibieron sulfato de magnesia. La aparición de convulsiones ocurrió en 6 de los 110 pacientes $(5.5 \%)$ en el grupo de la nimodipina Vs 1 de $108(0.9 \%)$ en el grupo del sulfato. La diferencia no fue estadísticamente significativa. Analizando los datos se observó que 4 de los pacientes del grupo de la nimodipina eran hipertensos crónicos y su tensión arterial no fue debidamente controlada con el fármaco. Se especula que el fenómeno de sobreperfusión vascular a nivel cerebral pudo 
haberse empeorado en este grupo de pacientes quienes persistieron hipertensas y vasodilatadas cerebralmente.

Actualmente se adelantan trabajos de investigación clínica con dosis de nimodipina de $60 \mathrm{mgs}$. cada 4 horas y uso simultáneo de otro antihipertensivo en pacientes con tensiones arteriales mayores a 160/110. Las conclusiones iniciales de los estudios sugieren :

- No existen diferencias significativas en la prevención de convulsiones entre los 2 grupos y la nimodipina parece ser una alternativa viable para la prevención de eclampsia.

- La nimodipina ofrece mayor y mejor control de la tensión arterial sin necesidad de agregar otros fármacos antihipertensivos, al compararlo con el sulfato.

- La nimodipina ofrece un mejor nivel de seguridad que el sulfato.

- La nimodipina es menos costosa y más segura que el sulfato; se administra vía oral sin necesitar un monitoreo tan cuidadoso como el sulfato dada su pobre asociación a complicaciones y efectos secundarios.

Si asumimos que su efectividad en la prevención de convulsiones es similar a la del sulfato, se convertiría en la droga de elección para la prevención y manejo de la eclampsia. Sin embargo el limitado número de casos estudiados hasta la fecha no permiten aseverar esto.
Los estudios mediante doppler de la circulación cerebral han especulado que existen 2 poblaciones de eclámpticas: Un primer grupo donde con cifras tensionales medianamente elevadas y cefalea intensa se convulsiona; el fenómeno se presume es de una vasoconstricción cerebral con isquemia. Un segundo grupo con tensión arterial marcadamente elevada como signo cardinal de la enfermedad, el problema parece ser una vasodilatación con sobreperfusión cerebral. En el primer grupo se asume una presión de perfusión cerebral reducida, caso en el cual la vasodilatación cerebral es más importante que el uso de un antihipertensivo.

Especulamos pues que en pacientes con preeclampsia severa y/o hipertensión arterial crónica y donde se supone existe una sobreperfusión, se prefiera el sulfato de magnesio como profiláctico anticonvulsivo. En pacientes con tensión arterial moderadamente elevada y sospecha de vasoconstricción cerebral, se utilice la nimodipina.

Quizás en un futuro cercano más estudios que abarquen un mayor número de pacientes confirmen las primeras evidencias de los beneficios de la nimodipina. Mientras tanto invitamos al lector a replantear los conceptos etiológicos tradicionales de la preeclampsia, así se logrará un mejor entendimiento de las alternativas terapéuticas que muy posiblemente se impondrán en los próximos años.

\section{BIBLIOGRAFIA}

1. Dumont A., Flahault A., Beaufils M. Effect of aspirin in pregnant women is dependent on increase in bleeding time. Am J Obstet Gynecol. Enero 1999;180: 135-40.

2. Sibai Baha M. Prevention of pre-eclampsia: A big disappointment. Am J Obstet Gynecol. Noviembre 1998; 179: 1275-8.

3. Herrera J., Arévalo-Herrera M. Prevention of preeclampsia by linoleic acid and calcium supplementation: A randomized controlled trial. Obstet Gynecol 1998; 91: 585-90.

4. Caritis S., Sibai B., Hauth J. Predictors of pre-eclampsia in women at high risk. Am J Obstet Gynecol. 1998; 179: 946-51.

5. Caritis S., Sibai B., Hauth J. Low-dose aspirin to prevent preeclampsia in women at high risk. The New England Journal of Medicine. Marzo 1998; 338 (11): 701-5.

6. Barth W. Low-dose aspirin for preeclampsia_ The unresolved question. Editorial. The New England Journal of Medicine. Marzo 1998; 338 (11): 756-7.

7. López-Jaramillo P., Delgado F. Calcium supplementation and the risk of preeclampsia in Ecuadorian pregnant teenagers. Obstet Gynecol 1997; 90: 162-7.

8. Sibai B., Ewell M., Klebanoff M. Risk factors associated with preeclampsia in healthy nulliparous women. Am J Obstet Gynecol. Noviembre 1997; 177: 1003-10.

9. Levine R., Hauth J., Curet L. Trial of calcium to prevent preeclampsia. The New England Journal of Medicine. Julio 1997; 337: $69-76$.
10. Witlin A, Sibai B. Hypertension in pregnancy: Current concepts of preeclampsia. Annu. Rev. Med. 1997; 48: 115-27.

11. Roberts J. Prevention or early treatment of preeclampsia. Editorial. The New England Journal of Medicine. 1997; 337: 124-5.

12. Bucher H., Guyatt G., Cook R. Effect of calcium supplementation on pregnancy-induced hypertension and preeclampsia. A metaanalysis of randomized controlled trials. JAMA. Abril 1996; 275: 1113-17.

13. Sánchez-Ramos L., Adair D., Kaunitz A. Calcium supplementation in mild preeclampsia remote from term: A randomized double-blind clinical trial. Obstet Gynecol 1995; 85:915-8.

14. Sánchez-Ramos L., Briones D., Kaunitz A. Prevention of pregnancy-induced hypertension by calcium supplementation in angiotensin II-sensitive patients. Obstet Gynecol 1994; 84: 349-53.

15. Sibai B., Caritis S., Thom E. Prevention of preeclampsia with lowdose aspirin in healthy, nulliparous pregnant women. The New England Journal of Medicine. 1993; 329: 1213-8.

16. Moya F., Germain A. Prevention of preeclampsia with low-dose aspirin. The New England Journal of Medicine. Marzo 1994; 330(11): 794-5.

17. Hauth J., Goldenberg R., Parker R. Low-dose aspirin therapy to prevent preeclampsia. Am J Obstet Gynecol 1993; 168: 1083-93. 